discovery was at once confirmed and applauded by others, including Benjamin Franklin.

Aepinus's work on tourmalin had been done in Rostock; soon thereafter he moved to St Petersburg and spent the remainder of his scientific life in the service of the Imperial Russian Academy of Sciences. In his book he remedied the following defects in the Franklinian theory of electricity: he got rid of Franklin's doctrine of electric "atmospheres", replacing it by the concept of electrical force acting at a distance; he explained how negatively charged bodies might attract one another by postulating that "common matter" deprived of its "normal" amount of electrical fluid will repel similar "common matter"; finally, he attempted to reduce electrical science to a set of mathematical principles, an endeavour that fell somewhat short of the mark since Aepinus did not know the inverse-square law of electrical (and of magnetic) attraction. $\mathrm{He}$ also constructed a parallel theory for magnetic forces and effects.

This first translation of Aepinus's essay, by P. J. Connor of the Department of Classics of the University of Melbourne, reads well and seems to be accurate. The introductory monograph, by R. W. Home of the same university, is a mine of information concerning the life and career of Aepinus, his influence, the state of electrical and magnetic experiment and theory in the eighteenth century, the organization of research in the Imperial Russian Academy of Sciences and much else. This monograph, it should be noted, is of book-length, just about as long as Aepinus's Tentamen, which it introduces.

The monograph and translation form a first-rate contribution to our knowledge of the history of science. Together they should help restore Aepinus to his proper place in the development of electricity and the use of mathematics in physical science.

I. B. Cohen is Professor in the Department of the History of Science, Harvard University, Cambridge, Massachusetts.

\section{Birds' sense of direction}

\section{John R. Krebs}

Avian Orientation and Navigation. By K. Schmidt-Koenig. Pp.180. (Academic: London and New York, 1979.) £13.80, $\$ 32$.

THIS is a well-written, balanced and accurate introduction to the literature on bird navigation and orientation up to the end of 1978. The major conclusions from work on pigeons are that birds find their way by using compasses based on the Sun and the Earth's magnetic field (nocturnal migrants also use the stars) in conjunction with an unidentified map. The map might well be based on magnetic information acquired by the pigeon during its outward journey to the release site, since pigeons transported in an altered magnetic field show less accurate homeward orientation when released. The suggestion by Italian workers that pigeons may sniff their way home by using a scent map is still controversial. One of the most convincing experiments was one in which pigeons in their home loft were exposed to clockwise or counterclockwise deflected winds. When released, these birds flew with a predicted deviation from the correct homeward direction. However, the same effect is shown by pigeons with anaesthetized olfactory epithelia, suggesting that information other than scent must be related to wind direction.

\section{Multivariate biology}

\section{Robert R. Sokal}

Morphometrics, The Multivariate Analysis of Biological Data. By Richard A. Pimentel. Pp.288. (Kendall/Hunt: Dubuque, Iowa, 1979.) Paperback \$19.95.

PRESENTING the results of morphometric, especially multivariate morphometric research, to a scientific audience is a major challenge. How can the reader make sense of numerous matrices, vectors and bivariate plots embellished with often unfamiliar terminology and esoteric mathematical and statistical manipulations? The author has set himself the task of remedying this situation by introducing biologists in a mathematically simple manner to the various multivariate techniques. To prevent misunderstandings I must point out at the outset that Pimentel uses the term morphometrics in an unconventional sense to include the application of multivariate analysis to unravel any pattern of variation including ecology, behaviour and physiology, not just the biometric analysis of phenotypic morphological variation in animals and plants.

The standard topics of multivariate analysis are covered in a very personal style with the author providing caveats about use of various methods and indicating quite clearly which he prefers. Multiple factor analysis is given short shrift, whereas discriminant analysis is given a very full, well-presented treatment. Although there are some examples of applications, I would have liked to see a specific section in which the methods are compared and the types of methods suitable for given biological questions are pointed out. In my judgement, multivariate morphometrics (in the narrow sense) is one of the most promising disciplines of evolutionary research. Yet so far a general discussion of this subject from a biological point of view has not appeared. Thus in addition to this useful volume we need yet another account - a discussion of the genetic, developmental and ecological implications of the multivariate assessment of morphological phenotypes.

Robert R. Sokal is Leading Professor of Ecology and Evolution at the State University of New York at Stony Brook.
One remarkable outcome of studies of homing is that pigeons have been shown to possess unsuspected sensory capabilities. Conditioning techniques have been used to show that pigeons can detect small changes in atmospheric pressure, infrasounds as low as $0.06 \mathrm{~Hz}$, ultraviolet light and the plane of polarized light. The demonstration of ultraviolet sensitivity is an interesting case history. Previous workers had noted that the sensitivity of pigeons decreased towards wavelengths of $400 \mathrm{~nm}$. However, below $400 \mathrm{~nm}$ sensitivity suddenly increases again to a second peak at $360-325 \mathrm{~nm}$. We still do not know what role these various sensory abilities play in navigation and orientation, but infrasound might be a good candidate for a large-scale auditory map, since very low frequency sounds generated by waves, wind in mountain ranges and so on may carry for several thousand kilometres. The discovery that birds, in common with many other animals, can detect the Earth's magnetic field is also the result of orientation studies. Schmidt-Koenig's book was completed just before the recent demonstration by Walcott et al. (Science 205, 1027-1029; 1979) that pigeons have tiny crystals of magnetite in their heads which could be part of the sense organ used to detect magnetic fields.

As with homing pigeons, more is known about how natural migrants use compasses than about their maps. Even relatively complex migration routes may be followed entirely with a compass. Garden warblers (Sylvia borin), for example, seem to have a built-in clock and compass which tells them to fly SW for one month in the autumn, followed by SSE for another month. This takes the birds from central Europe to southern Spain and thence to west or central Africa. Whether such birds have any map sense remains uncertain.

I have summarized a small sample of the results described by Schmidt-Koenig and I recommend that anyone interested in the subject should read his book.

John R. Krebs is a Lecturer in the Department of Zoology, University of Oxford, UK. 